Scientific Visualization, 2019, volume 11, number 5, pages 26 - 34, DOI: 10.26583/sv.11.5.03

\title{
Model, calculation method and visual representation of residual stresses in laser sintering of metal powders
}

\author{
A.V. Koldoba1,A, Yu.A. Poveschenko²,B, M.V. Popov3,C,D, R.V. Grishaev4,E, F.Kh. Mirzade5,E, \\ V.G. Niziev ${ }^{6, \mathrm{E}}$ \\ A Moscow Institute of Physics and Technology \\ B Keldysh Institute of Applied Mathematics RAS \\ C National Research Nuclear University MEPhI \\ D Polytech Lyon, Université Claude Bernard Lyon \\ E Institute on Laser and Information Technologies RAS \\ ${ }^{1}$ ORCID: 0000-0002-7509-4852 \\ 2 ORCID: 0000-0001-9211-9057, hecon@mail.ru \\ 3 ORCID: 0000-0002-7389-1081 \\ 4 ORCID: 0000-0003-1492-5576 \\ 5 ORCID: 0000-0003-4217-8109 \\ 6 ORCID: 0000-0003-3044-8394
}

\begin{abstract}
$\underline{\text { Abstract }}$
One of the key problems of materials science that arises in the technology of laser deposition of powders is the prediction of residual stresses in the product, which are formed as a result of local melting of the material and its subsequent relaxation to the initial temperature, accompanied by non-uniform solidification. A method for investigation of the stress-strain characteristics both in a weld bead and in a substrate due to local heating (above the melting temperature) and subsequent solidification in laser sintering of metal powders is suggested. The model includes two-dimensional stationary equations of thermoelasticity describing the stress-strain state of a non-uniformly heated product, as well as the relations approximating the thermophysical and elastic properties of the used materials in a wide temperature range, including the phase transformation (melting). The computational algorithm developed for the numerical integration of the thermoelasticity equations is based on the support operator method. The algorithm is implemented as a code and visual presentation of the results describing the residual stresses during laser sintering of metal powders for computers with parallel architecture. The joint flat technology was used to obtain the image of the fields of scalar quantities.This was done by filling the vector quantities shown by streamlines with color and the direction field. The spatial distribution of residual thermoelastic stresses in the substrate and in the deposited layer obtained as a result of numerical simulation allows to predict the defects appearing in the product during laser deposition of powders, which depend on the laser treatment mode and on the thermophysical properties of the materials.
\end{abstract} fer.

Keywords: Additive manufacturing, Metal microparticles, Laser sintering, Heat trans-

\section{Introduction}

Additive manufacturing technologies are currently fast progressing. Particular expectations are related with the production of parts by laser sintering / melting (LS) of metal powders [1]. The key issue here is the quality of the material of the final product. Among a number of difficulties in such technology, the problem of the residual stresses, appearing after material heating (local melting) and its subsequent cooling, is one of the most challenging. In a wide 
range of process parameters and used materials, there may be the cases where the residual stresses result in the defects, e.g. microcracks, and therefore the usage of the product under mechanical load becomes impossible.

There is no quite robust and precise manner to experimentally measure the residual thermal stresses during the process of cooling and solidification of the weld bead. Therefore, to solve this problem, as an addition to experimental research, it is crucial to apply mathematical modeling on high-performance computers of the heat and mass transfer during the phase transitions [2, 3], as well as the thermo-mechanical phenomena arised in the bead during its solidification.

With a variety of factors affecting the formation of residual stresses, and the complexity of the mathematical description, many issues of the problem of prediction and control of the stresses, theirs magnitudes and signs, are always under discussion. A mathematical model of a stress-strain state behavior of the weld bead must include the relation between the stress and the strain tensors in a wide temperature range, enclosing phase transitions. The temperature dependence of the elastic moduli, in particular the Young modulus, of the produced material is very essential here.

The existing models of formation of residual stresses, presented in [4-7], are valid in a narrow temperature range and do not take into account the temperature dependence of the elastic properties of the material. There are limitations in a simplified model [8] that do not allow to consider the results as complete and convincing. The model [8] takes into account the temperature dependence of the thermal expansion coefficient only, the temperature dependences of the elastic moduli are ignored. The applied approach to description of thermoelastic stresses at high temperatures is also questionable. Calculations are made without reference to the actual temperature fields and the form of the welding bead. In the paper [9], 3D numerical analysis of temperature and stress fields is performed on the basis of the temperature dependencies of elastic and thermophysical parameters of the material, such as the Young modulus, the thermal expansion coefficient, the yield stress. However, the mathematical description of the thermal problem in [9] is fragmentary and does not consider the kinetics of phase transitions.

The aim of this work is to build a physical and mathematical model, a numerical algorithm, and a visual presentation of the results describing the residual stresses in LS of metal powders under conditions more close to real ones. By data visualization using a joint flat technology for representing fields of scalar quantities by filling in the color and direction fields of vector quantities shown by streamlines, an effective stress analysis was performed for a specific mode of the LS process. The visualization tools used also make it possible, by analyzing residual stresses, to predict the appearance of microcracks that occur in the product after the laser powder deposition process, depending on the processing mode.

\section{Modelling of residual stresses distributions}

As a numerical analysis of the stress-strain state of the weld bead is carried out, let us consider the two separate tasks of the boundary-value problem of thermomechanics for crystallizing melt : thermal and thermoelastic according to the definition of stress-strain state, which are solved sequentially. As a result of solving the thermal problem, the temperature field and the position of the regions of solid and molten states of the deposited material and substrate are determined. The thermal problem in LS is described by the non-stationary heat equation taking into account the phase transition (Stefan problem) [6]. The solution to the thermal problem is then used as input to determine the stress-strain state of the roller and substrate. The modeling of the process of solidification of the melt and the occurrence of residual stresses, in turn, is carried out in two stages: the calculation of the stress-strain state of the unmelted part of the substrate at the stage of laser exposure and the calculation of the stress-strain state as the product cools to its initial (reference) temperature. 
Two typical temperatures can be determined for most metals, the melting temperature $T_{m}$ and the temperature $T_{S}<T_{m}$, at which the yield stress $\sigma_{S}$ of the material is close to zero. For further consideration it is practical to identify the zone where the material loses its elastic properties. Its boundaries are somewhat wider than the boundaries of the liquid phase and are determined by the isotherm ${ }^{T_{S}}$. In a region with ${ }^{T>T_{S}}$ the stresses are considered zero.

In that part of the substrate, where ${ }^{T<T_{S}}$ stationary Lame equations should be solved. Elastic modules included in these equations are considered to be dependent on the temperature via the temperature dependence of the Young's modulus. The cause of the elastic stresses is thermal expansion of the material. The thermal expansion magnitude is governed by the local temperature. The temperature field is considered as known (from calculations on previous stages of the laser radiation impact on the substrate and the welded on it powder). At the first stage (heating from the reference temperature $T_{a}$ to ${ }^{(y, z)}$ ) the equations of mechanical equilibrium are considered:

$$
\begin{gathered}
\frac{\partial \sigma_{i k}^{\prime}}{\partial x_{i k}}=0 \\
\sigma_{i k}^{\prime}=2 \mu \varepsilon_{i k}^{\prime}+\lambda \varepsilon^{\prime} \delta_{i k}-\alpha(2 \mu+3 \lambda)\left(T-T_{a}\right) \delta_{i k}, \\
\varepsilon_{i k}^{\prime}=\frac{1}{2}\left(\frac{\partial u_{i}^{\prime}}{\partial x_{k}}+\frac{\partial u_{k}^{\prime}}{\partial x_{i}}\right),
\end{gathered}
$$

where $\sigma_{i \bar{k}}^{\prime}$ is the full stress tensor; $\varepsilon_{i k}^{\prime}$ is the strain tensor; $\varepsilon^{\prime}=$ div $\mathbf{u}^{\prime}$ is deformation of the material; ${ }^{u_{i}^{\prime}}$ are the components of the displacement vector ${ }^{\mathbf{u}^{\prime}}$ of the material; ${ }^{\mu}, \lambda$ are the elastic moduli (Lame coefficients); $\alpha$ is the thermal expansion coefficient; $T_{a}=20^{\circ} \mathrm{C}$ is the reference temperature; the indices $i, k=x, y, z$.

We assume that evolution occurs via a series of plane strain states. This means that there are no displacements of the material along the direction of the laser beam movement (i.e. $x_{-}$ direction) at each moment. In this case the problem becomes two-dimensional and $\varepsilon_{x x}=\varepsilon_{x y}=\varepsilon_{x z}=0$. The non-zero strain tensor components are related with the components of displacements by the following relations:

$$
\begin{gathered}
\varepsilon_{y y}=\frac{\partial u_{y}}{\partial y}, \quad \varepsilon_{z z}=\frac{\partial u_{z}}{\partial z} \quad \varepsilon_{y z}=\frac{1}{2}\left(\frac{\partial u_{y}}{\partial z}+\frac{\partial u_{z}}{\partial y}\right), \\
\varepsilon=\varepsilon_{y y}+\varepsilon_{z z} .
\end{gathered}
$$

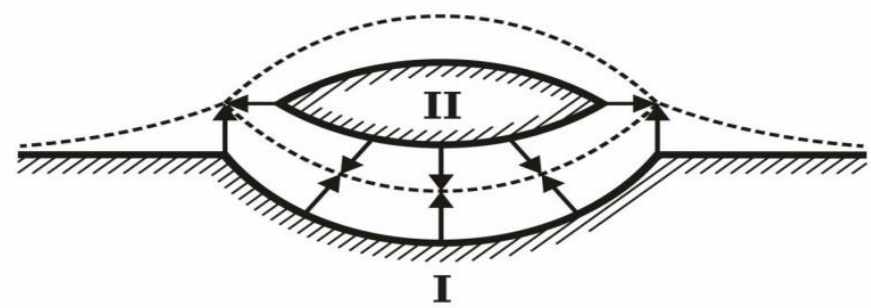

Figure 1: Schematic representation of the two-step algorithm for residual stresses calculations. 
The problem is solved in the domain, composed of two subdomains (Fig. 1). A subdomain I contains the material at the temperature ${ }^{T<T_{S}}$. In a subdomain II the temperature is $T>T_{S}$. The subdomain II is not considered, as it is assumed that there are no stresses in it. The thermal stresses in the subregion I lead to area stretching relative to the reference state at temperature $^{T_{a}}$. Subregions I and II are joined (have no gaps and overlaps) and have a temperature distribution calculated at the first stage of modeling.

In Fig. 1 the shaded areas I and II represent the material of the substrate and the bead under the reference conditions (not heated or strained). Heated up to the first stage temperature, the material expands and fills a region those boundaries are shown by the dashed lines. In this state the subdomains I and II densely adjoin to each other.

The material, returning to its initial reference temperature, would return to the reference state with the gap between the subdomains. But since the actual gaps and overlapping do not exist after cooling to the reference temperature, the subdomains can not return to their initial states free from the stresses. In other words, the material of the substrate and the weld should contain residual stresses.

At the lower boundary of the computational domain we apply the Dirichlet boundary condition for the displacements $u_{i}^{\prime}=0$. At the other boundaries we apply the Newmann boundary condition ${ }^{n_{k}} \sigma_{i k}^{\prime}=0$, i.e. the absence of the normal to the boundary total stresses.

The equations contain the elastic modulus ${ }^{\lambda}$ and the shear modulus ${ }^{\mu}$. These quantities depend linearly on the Young's modulus $E(T)$ as

$$
\lambda(T)=\frac{v E(T)}{(1+v)(1-2 v)}, \quad \mu(T)=\frac{E(T)}{2(1+v)} .
$$

The temperature dependence is introduced into the Young's modulus as $E(T)=E_{a} \phi(T)$. Here $E_{a}=7 \times 10^{11} \mathrm{dyn} / \mathrm{cm} 2$ is the Young's modulus at the reference temperature $T_{a}$; Poisson's ratio $v=0.36$ is considered temperature-independent. As a specific example, the temperature dependence of the Young's modulus is taken in the following calculations as [10]:

$$
\phi(T)=\left\{\begin{array}{c}
1.03\left(1-\frac{T}{2 T_{m}}\right), \quad T<T_{*}, \\
1.03\left(1-\frac{T}{2 T_{m}}\right) e^{-0.05\left(T-T_{*}\right)}, \quad T>T_{*},
\end{array}\right.
$$

where $T_{m}=857^{\circ} \mathrm{K}$ is the melting temperature.

The temperature $T_{*}=650^{\circ} \mathrm{K}$ is chosen as it provides the best agreement between the empirical relation and experimental measurements. It is assumed that the thermal expansion coefficient does not depend on the temperature and is equal to $\alpha=2.2 \times 10^{-7} \mathrm{~K}^{-1}$.

At the second stage of the simulation it is assumed that the temperature in the whole region is reduced to its reference value ${ }^{T_{a}}$. At the same time due to the differences in strains in the "melted" and "unmelted" subdomains, the residual stresses appear. At this stage the equation (1) is solved numerically in both subdomains I and II. As $T=T_{a}=$ const , the thermal stresses are absent. In the same way as it is at the first stage of simulation, at the lower boundary of the joint computational domain I+II the Derichlet's boundary condition $u_{i}^{\prime}=0$ for the displacements is applied. On the other boundaries the Newmann's boundary condition $n_{k} \sigma_{i k}^{\prime}=0$ is applied. 
The problem (1) is solved for the displacements. The temperature field and the thermal term are assumed to be defined. At the first stage the displacements of the elastic material particles are calculated.

After the temperature in the both regions returns to the initial reference value at fixed particle displacements, obtained at the first stage. In order to the particles do not shift from now on, they must be under the action of "virtual" force with density

$$
f_{i}=-\frac{\partial \sigma_{i k}}{\partial x_{k}} \quad, \quad \sigma_{i k}=2 \mu \varepsilon_{i k}^{\prime}+\lambda \varepsilon^{\prime} \delta_{i k}, \quad \varepsilon_{i k}=\frac{1}{2}\left(\frac{\partial u_{i}^{\prime}}{\partial x_{k}}+\frac{\partial u_{k}^{\prime}}{\partial x_{i}}\right),
$$

where the elastic moduli are calculated at the temperature ${ }^{T_{a}} ; u_{i}^{\prime}$ are the displacements obtained at the first stage.

Note that in the subdomain II the "virtual" force acts only on the surface and provides a uniform tension of this subdomain, in accordance with the thermal expansion obtained at the first stage.

At the second stage the "virtual" force disappears, and the particles of the elastic material, enclosed within the subdomains I and II, relax to the "natural" state. This state, however, does not coincide with the initial one for the reasons mentioned above. At this point, the following problem is considered

$$
\sigma_{i k}=2 \mu \varepsilon_{i k}+\lambda \varepsilon \delta_{i k}, \quad \varepsilon_{i k}=\frac{1}{2}\left(\frac{\partial u_{i}}{\partial x_{k}}+\frac{\partial u_{k}}{\partial x_{i}}\right),
$$

The displacements of the material particles (after the stages "heating+melting" and "solidification+cooling”) obtain finally the following values

$$
u_{i}=u_{i}^{\prime}+u_{i}^{\prime \prime} \neq 0 \text {. }
$$

The residual stresses are calculated for these residual displacements by the formula (2), where the elastic moduli are calculated at the reference temperature $T_{a}$.

The output data for the proposed mathematical model are temperature fields, components of the stress tensor (as well as the main directions and stresses calculated from them), components of the displacement vector. For greater clarity, when analyzing the results of numerical modeling, it is proposed to use a joint flat technology as representation of the fields of scalar quantities. This is done by filling the vector quantities shown by streamlines with color and the direction field. To implement this visual presentation method, the well-known graphic visualization package TecPlot [11] and the corresponding data file format were used.

\section{The results of simulation.}

The difference scheme that approximates the Lame equations on a curvilinear mesh is based on the support operators method [12]. The method provides self-adjointness and positivity of an approximation difference operator, if the parent operator of the initial differential problem has the same properties. The matrix of the set of linear equations for the displacements is inversed by conjugate gradient method.

Calculations were performed for silumin powder. The laser radiation power is $P=3 \mathrm{~kW}$, the laser beam radius is $1.67 \mathrm{~mm}$, the laser light absorption factor is 0.45 , the radius of the powder jet is $4 \mathrm{~mm}$, the capture efficiency of powder is 0.7 , the mass discharge of the powder is $20 \mathrm{~g} / \mathrm{min}$.

The temperature distribution in the substrate at the heating stage is presented in Fig. 2. Here, the lines indicate the direction of the displacements. Fig. 3 shows the distribution of the main stresses in the substrate at the heating stage. The lines indicate the direction the main stresses, color represents the corresponding values.

The distribution of the residual displacements in the substrate and the weld bead after cooling is shown in Fig. 4. Fig. 5 shows the distribution of the main stresses in the substrate and 
the weld bead after cooling. The lines indicate the direction of the main stresses, color represents the corresponding values.

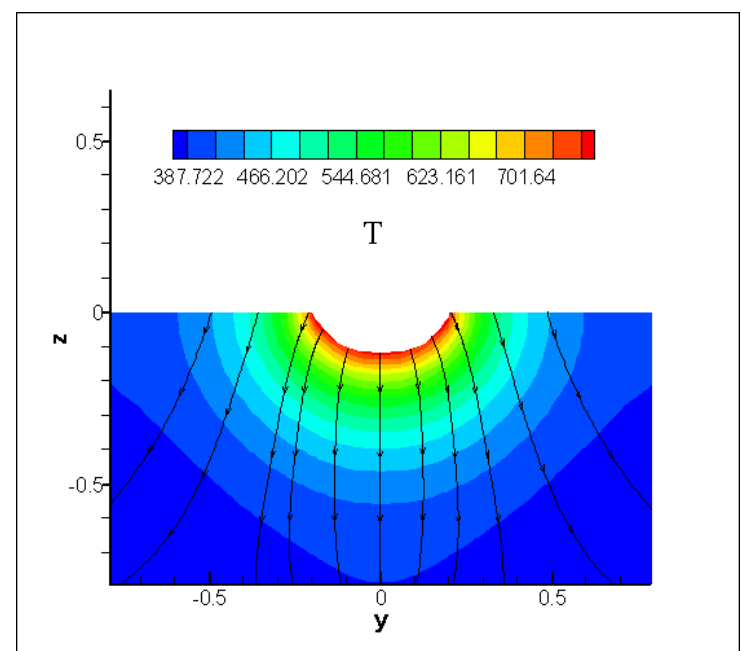

Figure 2: The temperature distribution, established in the substrate at the heating stage. The lines show the direction of the displacements.
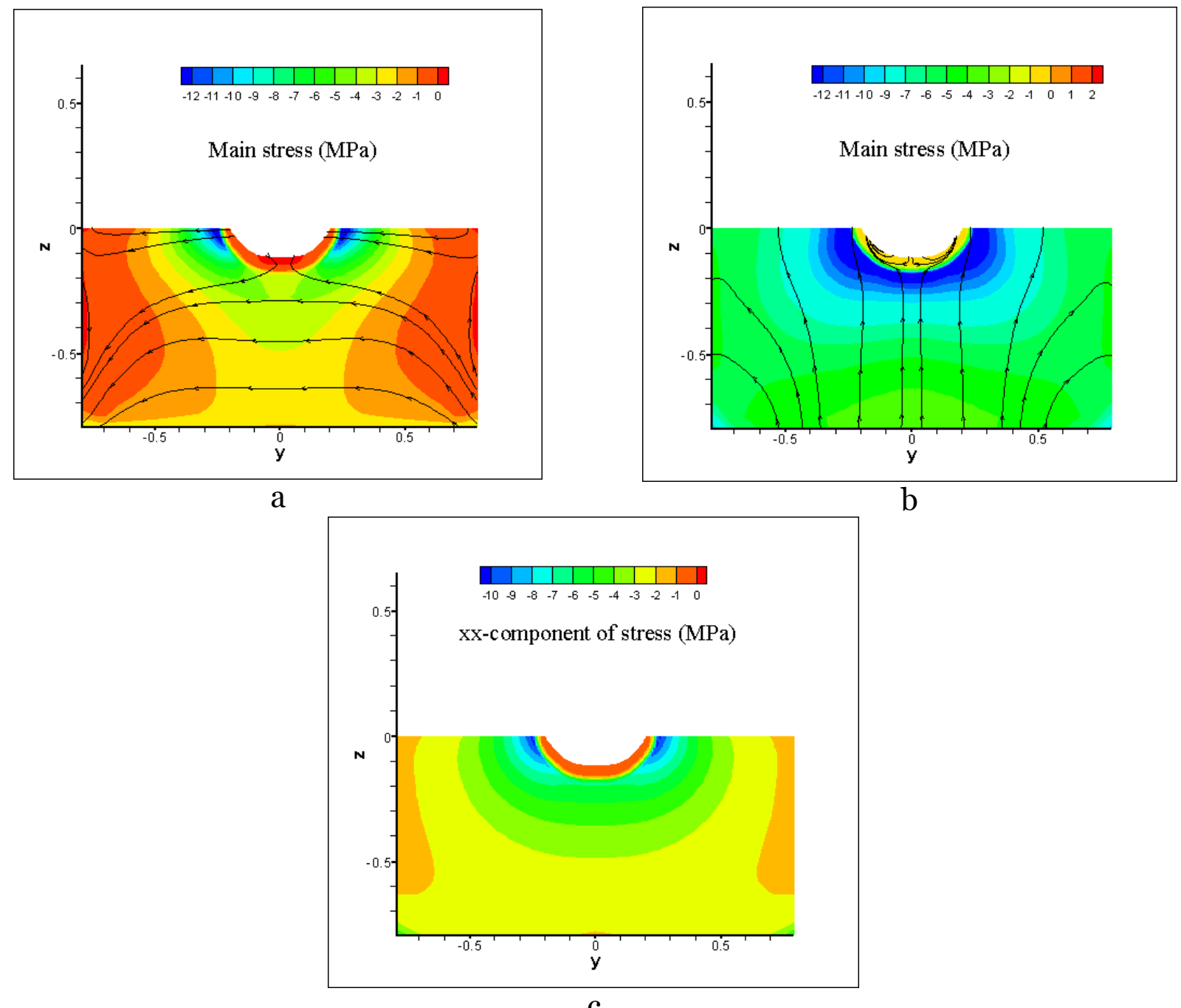

Figure 3: Distributions of main stresses in the substrate at the heating stage. The lines indicate the direction of the stresses, color represents the corresponding values (MPa). Cases A, $\mathrm{B}$ : the stresses at the section y-z. Case C: the same along the scanning direction of the laser beam (x-axis). 


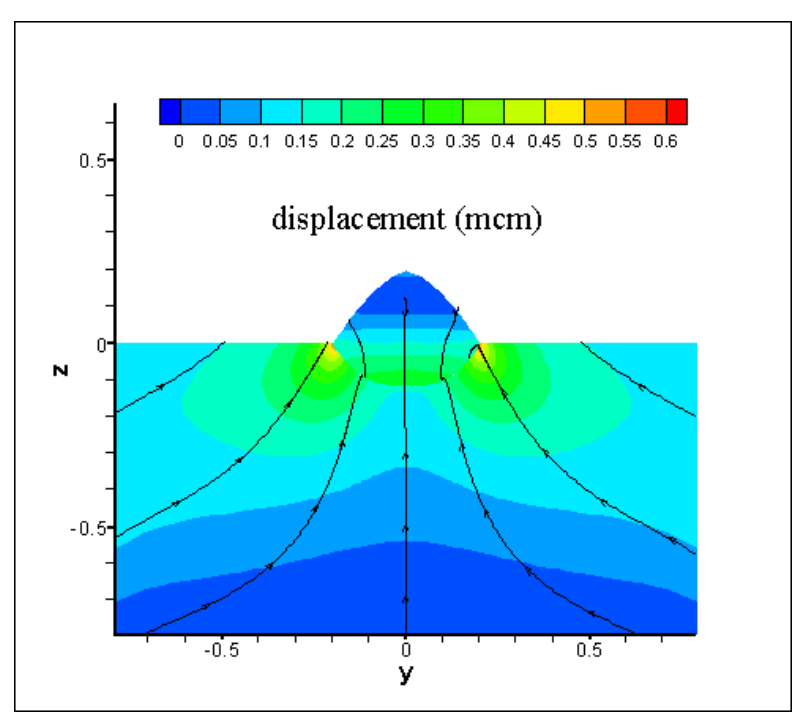

Figure 4. Distribution of residual displacements $(\mathrm{mcm})$ in the substrate and the weld bead at the cooling stage. The lines show the direction of the displacements, color represents the corresponding values in microns.

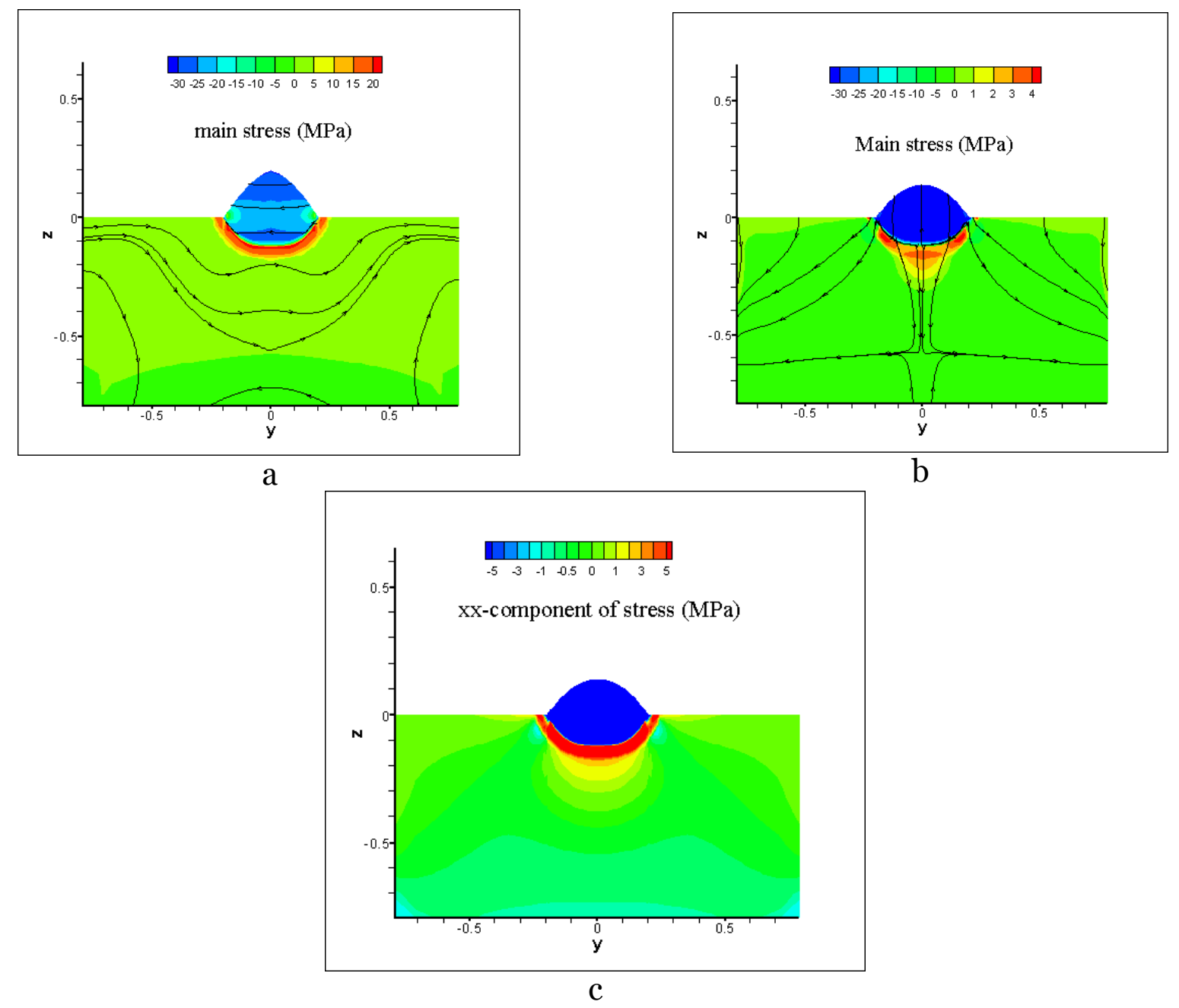

Figure 5: Distributions of main stresses in the substrate and the weld bead at the cooling stage. The lines indicate the direction of the stresses, color represents the corresponding values (MPa). Cases A, B: the stresses at the section y-z. Case C: the same along the scanning direction of the laser beam (x-axis). 


\section{Conclusions}

Study the stress-strain state that occurs in LS of metal in weld bead and substrate, a mathematical model and the corresponding computational algorithm are developed. A technique is proposed for visual presentation of the results of numerical simulation of the stress-strain state (deformation and stress fields) arising from local heating, partial melting and subsequent cooling of the product. Using the developed computer technology, the stress fields were calculated and visualized for a specific mode of the LS process. It was found that in the boundary layers of the weld layer, the stress level is the highest. Perhaps this is due to large temperature gradients in these zones. In numerical experiments, it was shown that taking the temperature dependence of Young's modulus into account when determining the stressstrain state in products leads to a decrease in the calculated stress levels. In addition, taking this dependence into account can lead to noticeable qualitative differences in the pattern of stress state evolution compared with the elastic setting. The resulting patterns of residual stresses make it possible to predict the appearance of microcracks arising in the product after the process of laser powder deposition, depending on the processing mode. The presented numerical model can be used to calculate the residual stresses of a real process in the presence of physical parameters of the material.

\section{References}

1. I. V. Shishkovsky. Laser Synthesis of Functional Mesostructures and 3D Parts.

2. V. G. Niz'ev, F. Kh. Mirzade, V. Ya. Panchenko, V. M. Chechetkin, and G. V. Ustiugova. Heat and mass transfer under laser sintering of a powder mixture. Mathematical Models and Computer Simulations, 4(2):163-171, 2012.

3. V. G. Niz'ev, F. Kh. Mirzade, and V. Ya. Panchenko. Numerical modeling of laser sintering metallic powders // Collective monograph: Modern laser and information technologies.

4. F. Brückner, D. Lepski, and E. Beyer. Modeling the Influence of Process Parameters and Additional Heat Sources on Residual Stresses in Laser Cladding. Journal of Thermal Spray Technology, 16:355-373, September 2007.

5. R. Jendrzejewski, G.' Sliwi' nski, M. Krawczuk, andW. Ostachowicz. Temperature and stress fields induced during laser cladding. Computers \& Structures, 82(7-8):653-658, 2004.

6. V. Ocelík, J. Bosgra, and J.Th.M. de Hosson. In-situ strain observation in high power laser cladding. Surface and Coatings Technology, 203(20-21):3189-3196, 2009.

7. A. V. Gusarov, M. Pavlov, and I. Smurov. Residual Stresses at Laser Surface Remelting and Additive Manufacturing. Physics Procedia, 12:248-254, 2011.

8. A. V. Gusarov, I. S. Malakhova-Ziablova, and M. D. Pavlov. Thermoelastic Residual Stresses and Deformations at Laser Treatment. Physics Procedia, 41:896-903, 2013.

9. L. Hua,W. Tian,W. Liao, and C. Zeng. Numerical Simulation of Temperature Field and Residual Stress Distribution for Laser Cladding Remanufacturing. Advances in Mechanical Engineering, 6:id291615, 2014.

10. J.F. Bell and C. Truesdell. Mechanics of Solids: Volume I: The Experimental Foundations of Solid Mechanics. Springer Berlin Heidelberg, 1984.

11. TecPlot - CFD Visualization \& Analysis Software URL: https://www.tecplot.com/

12. A. V. Koldoba, Yu. A. Poveshchenko, I. V. Gasilova, and E. Yu. Dorofeeva. Numerical schemes of the support operators method for elasticity theory equations. Mathematical Modelling, 24:86-96, 2012. 